\title{
THE INHOMOGENEOUS MINIMA OF INDEFINITE QUADRATIG FORMS
}

\author{
E. S. BARNES
}

(received 5 September 1960)

If $f(x)$ is a real indefinite quadratic form in $n$ variables with determinant $d \neq 0$, we set for any real $\alpha$

$$
\begin{aligned}
& m(f ; \boldsymbol{\alpha})=\inf |f(\boldsymbol{x})| \quad \text { over } \boldsymbol{x} \equiv \boldsymbol{\alpha}(\bmod 1), \\
& m(f)=\sup _{\boldsymbol{\alpha}} m(f ; \boldsymbol{\alpha}) .
\end{aligned}
$$

A current problem in the geometry of numbers is the determination of bounds for the ratio $m(f) /|d|^{1 / n}$.

Setting

$$
c_{n}=\inf _{f} \frac{m(f)}{|d|^{1 / n}}
$$

Davenport [3] showed that $c_{2}>0$ (see Cassels [2] for a discussion of the literature on $c_{2}$ ). Cassels [2, p. 306] remarks that it is not known whether $c_{3}>0$.

It is in fact quite simple to show that

$$
c_{n}=0 \text { for } n \geqq 3 .
$$

I establish here the sharper result:

Theorem. For any $n \geqq 3$ and any signature $(r, s)(r \geqq 1, s \geqq 1, r+s=n)$, there exists a quadratic form $f$ of signature $(r, s)$ and determinant \pm 1 with $m(f)=0$.

The result in fact holds for any $f$ which represents arbitrarily small nonzero values for integral values of the variables.

We need the following lemma:

Suppose that $f$ has signature $(r, s)$ with $r \geqq 2, s \geqq 1, r+s=n$ and determinant $d \neq 0$, and that $f$ properly represents a value $a>0$; then, for some $\gamma>0$ depending only on $n$,

$$
m(f) \leqq\left(\gamma a^{n-2}|d|\right)^{1 /(2(n-1))}+\frac{1}{4} a .
$$

Proof. We may suppose, after a suitable equivalence transformation, that $f(1,0, \cdots, 0)=a$; we may then write 


$$
f(x)=a\left(x_{1}+\lambda_{2} x_{2}+\cdots+\lambda_{n} x_{n}\right)^{2}-g\left(x_{2}, \cdots, x_{n}\right)
$$

where $g$ is indefinite with determinant $-d / a$.

For any $\alpha$, we may choose $x_{j} \equiv \alpha_{j}(\bmod 1)(j=2, \cdots, n)$ so that

with

$$
g\left(x_{2}, \cdots, x_{n}\right)=b
$$

$$
0 \leqq b \leqq\left(\gamma \frac{|d|}{a}\right)^{1 /(n-1)} ;
$$

this is a particular case of a result of Blaney [1] (Theorem 2). With this choice of $x_{2}, \cdots, x_{n}$, we have

$$
f(x)=a\left(x_{1}+\lambda\right)^{2}-b .
$$

We now choose $x_{1} \equiv \alpha_{1}(\bmod 1)$ so that

$$
\left(\frac{b}{a}\right)^{\frac{1}{2}}-\frac{1}{2} \leqq x_{1}+\lambda<\left(\frac{b}{a}\right)^{\frac{1}{2}}+\frac{1}{2}
$$

an elementary calculation now gives

$$
-(a b)^{\frac{1}{2}}+\frac{1}{4} a \leqq f(x)<(a b)^{\frac{1}{2}}+\frac{1}{4} a .
$$

Thus, using (3), we have

$$
m(f ; \alpha) \leqq(a b)^{\frac{1}{2}}+\frac{1}{4} a \leqq\left(\gamma a^{n-2}|d|\right)^{1 /(2(n-1))}+\frac{1}{4} a ;
$$

since this holds for all $\alpha$, (1) follows immediately.

Proof of the Theorem. Take $n \geqq \mathbf{3}$ and $f$ any indefinite form of signature $(r, s)$, with determinant \pm 1 , which represents arbitrarily small non-zero values of each sign. (An example of such a form is

$$
\theta x_{1}^{2}-\frac{1}{\theta} x_{2}^{2}+x_{3}^{2}+\cdots+x_{r+1}^{2}-x_{r+2}^{2}-\cdots-x_{n}^{2},
$$

where $\theta$ has a continued fraction with unbounded partial quotients.) Since $m(-f)=m(f)$, we may suppose that $r \geqq 2$; then, by the lemma,

$$
m(f) \leqq\left(\gamma a^{n-2}\right)^{1 /(2(n-1))}+\frac{1}{4} a
$$

for any $a>0$ represented by $f$. Since $n \geqq 3$ and $a$ may be chosen as small as we please, it follows that $m(t)=0$.

This establishes the theorem, and hence the assertion (1).

\section{References}

[1] Blaney, H. Indefinite quadratic forms in $n$ variables, J. London Math. Soc. 23 (1948), $153-160$.

[2] Cassels, J. W. S. An introduction to the geometry of numbers (Springer, 1959).

[3] Davenport, $H$. Indefinite quadratic forms and Euclid's algorithm in real quadratic fields, Proc. London Math. Soc. (2) 53 (1951), 65-82.

University of Adelaide. 